\title{
Aprendizaje socioemocional en estudiantes de quinto y sexto grado: presentación y evaluación de impacto del programa BASE
}

\author{
Neva Milicic* \\ Lidia Alcalay** \\ Christian Berger*** \\ Pilar Álamos****
}

\section{Resumen}

Se presenta el desarrollo y evaluación de impacto de un programa para favorecer el bienestar y aprendizaje socioemocional en estudiantes de quinto y sexto año de enseñanza general básica: el programa BASE. A partir de una revisión teórica del aprendizaje socioemocional se fundamenta el énfasis del programa en el desarrollo de vínculos interpersonales de calidad y el desarrollo de las competencias necesarias para ello, integrando tanto factores a nivel del desarrollo individual como también del contexto que favorece este desarrollo, así como la generación de comunidades sanas y nutritivas. Se utilizó un diseño cuasi-experimental pre-post con grupo control para evaluar el impacto del programa. Los resultados muestran un impacto significativo positivo del programa en la autoestima, integración social, y percepción del clima escolar de los estudiantes que participaron de él en comparación al grupo control. Se discuten los resultados del programa y sus posibles implicancias para el desarrollo de intervenciones en este campo. Palabras clave: Aprendizaje socioemocional. Bienestar. Enseñanza general básica.

\section{Introducción}

Recientemente, un estudio que integró la evaluación de más de 200 programas orientados a favorecer el aprendizaje socioemocional en estudiantes escolares (DURLAK et al. 2011) vino a consolidar la ya creciente evidencia sobre el impacto de este tipo de intervenciones tanto para indicadores socioafectivos, como también para indicadores académicos. Este estudio, a través de un trabajo meta-analítico, demostró que los estudiantes que

\footnotetext{
* PhD. Pontificia Universidad Católica de Chile. E-mail: nmilicic@uc.cl

** MS, Pontificia Universidad Católica de Chile. E-mail: Ialcalay@uc.cl

*** PhD., Pontificia Universidad Católica de Chile. E-mail: cberger@uc.cl

**** MS(c), Pontificia Universidad Católica de Chile. E-mail: pmalamos@uc.cl
} 
participaron de dichos programas aumentaron sus competencias socioemocionales, sus actitudes y conductas prosociales, y subieron en 11 puntos porcentuales su rendimiento académico. Los resultados alcanzados por estos programas se fundamentan en diversos modelos teóricos y estudios previos que han mostrado la relevancia de la dimensión socioemocional para el bienestar y desarrollo positivo. Por ejemplo, mayores niveles de desarrollo socioemocional han sido asociados a mayores indicadores de bienestar general (EISENBERG, 2006; GUERRA; BRADSHAW, 2008), menor sintomatología depresiva (GOLDENBERG et al., 2006), y un mayor número relaciones interpersonales satisfactorias (LOPES et al., 2005). Estos efectos también se observan en población escolar y se transfieren a indicadores académicos. Así, recientes estudios muestran una asociación entre aspectos socio-emocionales y distintos indicadores académicos, como rendimiento (BERGER et al., 2011; EXTREMERA; FERNÁNDEZ-BERROCAL, 2003; STIPEK; MILES, 2008), aprendizaje y dominio de contenidos y habilidad lectora (ASHDOWN; BERNARD, 2012; HAWKINS et al., 1999), motivación y compromiso (MALECKI; ELLIOT, 2002).

Estos resultados hacen evidente la necesidad de intencionar la formación socioemocional de los estudiantes, pero al mismo tiempo ponen en el centro de la discusión las distintas nociones y aproximaciones existentes a dicha dimensión. La perspectiva de la inteligencia emocional (SALOVEY; MAYER, 1990) podría considerarse el paradigma que abrió la discusión sobre lo socioafectivo en la escuela. Sin embargo, para ello debió tomar la estructura de desarrollo cognitivo propia de los modelos de inteligencia, situación que con el tiempo ha ido revisándose y complementándose. En esta línea se ha ido consolidando la noción de aprendizaje socioemocional (ALCALAY et al., 2012). La perspectiva del aprendizaje socioemocional (HOFFMAN, 2009) ha complementado el foco exclusivo en un nivel individual de desarrollo socioemocional, integrando también el contexto interpersonal e institucional en el cual cada individuo se desarrolla (ZINS et al., 2004). Desde este enfoque se ha hecho énfasis en como el individuo genera vínculos interpersonales en contextos específicos, identificando factores favorecedores y obstaculizadores para el establecimiento de relaciones interpersonales nutritivas. Asimismo, se evalúa la manera en que cada individuo afecta tanto a los ambientes en los cuales participa, como a otros individuos que participan de dichos ambientes. Así, el foco puesto en el bienestar socioemocional implica una mirada no sólo a las competencias sino a otros aspectos como la valoración de sí mismo, el establecimiento de vínculos nutritivos con otros, y una percepción positiva del ambiente (BERGER et al., 2011). 
El trabajo sobre la dimensión individual del aprendizaje socioemocional ha sido abordado extensamente a través de distintos enfoques en las últimas décadas. Por ejemplo, los trabajos sobre autoestima (HAEUSSLER; MILICIC, 1995), en habilidades sociales (GRESHAM; ELLIOTT, 1984; HUBBARD; COIE, 1994), las competencias asociadas a la inteligencia emocional (BISQUERRA; PÉREZ, 2007; EXTREMERA; FERNÁNDEZ-BERROCAL, 2003; GOLEMAN, 1996) y estudios sobre estrategias de resolución de conflictos (EISENBERG; GARVEY, 1981; LAURSEN; FINKELSTEIN; TOWNSENDS, 2001). Si bien estos estudios son concluyentes respecto de la importancia de desarrollar competencias socioemocionales en el individuo, modelos actuales enfatizan la necesidad de generar ambientes escolares en los cuales los estudiantes se sientan seguros, valorados y apoyados, ambientes caracterizados por salas de clases cálidas y acogedoras, pero al mismo tiempo desafiantes (HAMM et al., 2011). En otras palabras, contextos que por una parte potencien y estimulen el desarrollo socioemocional, pero al mismo tiempo que no pierdan de vista la importancia de mantener una comunidad integrada en la cual el desarrollo y bienestar individual no se concibe sin un desarrollo y bienestar de todos los demás miembros de dicha comunidad, y por ende, de la sociedad en su conjunto (ALCALAY et al., 2012; CHAUX et al., 2004). Esto es especialmente relevante considerando el énfasis en la riqueza de la diversidad (étnica, religiosa, etc.) que ha cobrado fuerza en los últimos años, pero que al mismo tiempo implica una mayor complejidad.

Desde una perspectiva evolutiva lo anterior es consistente con la teoría del apego, enfatizando que profesores cálidos, contenedores y apoyadores proveen a los estudiantes con un sentido de conexión con el entorno escolar y de seguridad para explorar nuevas ideas y asumir riesgos - ambos fundamentales para el aprendizaje (MURRAY; GREENBERG, 2000). Más aún, estudios muestran como vínculos positivos con figuras significativas a lo largo de la vida permiten reparar patrones relaciones disfuncionales generados en etapas previas, lo cual enfatiza aún más la relevancia de las relaciones afectivas durante la etapa escolar (BERGIN; BERGIN, 2009). Desde esta perspectiva puede hablarse de apego escolar, destacando el rol de relaciones de cuidado y protección con adultos significativos en el contexto escolar que favorezcan el bienestar de los estudiantes (BERGER et al., 2009; LUCKNER; PIANTA, 2011; POTOCNJAK et al., 2011). Estos vínculos permitirían a los estudiantes procesar y elaborar su experiencia escolar con todos los desafíos que ella implica, favoreciendo la transmisión de una actitud positiva y de disfrute respecto de la vivencia escolar (FRENZEL et 
al., 2009), la cual redundaría en la motivación y autonomía de los estudiantes (ROTH et al., 2009). De hecho, estudios muestran el impacto de relaciones positivas entre estudiantes y sus profesores en la conexión de los alumnos con la institución, y en los resultados deseados para los estudiantes, tanto académicos como socioemocionales (ABBOTT et al., 1998; OSHER et al., 2007), impacto que se mantendría en sus vidas adultas (BIRCH; LADD, 1998; HAMRE; PIANTA, 2001, 2006; MURRAY; GREENBERG, 2000; PIANTA et al., 2003).

Si bien lo anterior enfatiza los vínculos entre estudiantes y profesores, los vínculos entre pares son también de gran relevancia para el desarrollo socioemocional, y cobran un rol particular durante la adolescencia, en donde el establecimiento de relaciones de intimidad y cercanía (OJANEN et al., 2005) y la participación y aceptación por parte del grupo (FLETCHER et al., 2006) son aspectos centrales de la experiencia social y emocional adolescente. Diversos autores muestran que adolescentes que forman parte de grupos cohesionados muestran mayores indicadores de logro académico y de adaptación y funcionamiento social (CHEN et al., 2008).

La revisión de los vínculos que establecen los estudiantes, tanto con sus profesores como con sus pares, junto con la manera en que se relacionan con la institución escolar en su conjunto, hacen parte de lo que diversos autores conceptualizan como clima social escolar (ARÓN; MILICIC, 1999). El clima social escolar considera el ajuste socioemocional y académico, la relación entre la experiencia escolar y problemas conductuales y ansiosos o depresivos y la adaptación social (KUPERMINC et al., 2001; KORNBLIT et al., 2008; LOUKAS; ROBINSON, 2004).

Integrando lo hasta aquí expuesto, resulta evidente que el bienestar socioemocional de los estudiantes, así como el desarrollo de competencias socioemocionales, no es un proceso exclusivamente individual sino que se desarrolla también en función del contexto social escolar, y por lo tanto debe ser intencionado a nivel local por las instituciones educativas, y a nivel global por las políticas públicas y los planes y programas de formación que los organismos centrales entregan a las instituciones.

\section{Programas de Aprendizaje Socio Emocional}

Evaluaciones de impacto de los programas orientados a favorecer el aprendizaje socioemocional muestran consistentemente un incremento en las habilidades sociales y emocionales de los estudiantes, en el sentimiento de pertenencia, en el desarrollo 
de conductas prosociales. Asimismo, muestran una disminución en la prevalencia de problemas conductuales (DURLAK; WEISSBERG, 2007; DURLAK et al., 2010; HAWKINS et al., 2004; PAYTON et al., 2000; ZINS et al., 2004).

Progresivamente estos programas han ido conceptualizando el desarrollo afectivo y social de una manera más integradora, incluyendo simultáneamente las diferentes áreas que en programas anteriores se habían abordado en forma separada y de manera específica. Asimismo, en la última década se han reportado diversas evaluaciones de estos programas, entregando evidencia y soporte empírico para su implementación (ÁLVAREZ, 2001). Estos resultados, sin embargo, no implican que los programas sean en sí la solución a problemas de convivencia, conflicto, y/o violencia, ya que un factor clave para su éxito es la capacidad que tengan de ser sensibles a las realidades locales y que sean percibidos como significativos por los actores de las instituciones locales (SMITH et al., 2003). El proceso de adaptación y validación de algún programa existente constituye un proceso largo y costoso que debe tomar en cuenta las creencias específicas que pueden originarse en la socialización recibida por los niños en los contextos escolares y familiares, así como aquellos aspectos que son idiosincráticos de la realidad en la cual pretende implementarse (HAWKINS et al., 2004). En base a lo anterior, se optó por desarrollar un programa de aprendizaje socioemocional que fuese efectivo y pertinente para la realidad chilena, apoyado en la evidencia internacional, pero también en un proceso de levantamiento de información local sobre las necesidades y particularidades de la población objetivo del programa (BERGER et al., 2009; RIVERA; MILICIC, 2005).

\section{Programa para el Bienestar y Aprendizaje Socio Emocional BASE}

El programa BASE para 5to y 6to año de enseñanza general básica constituye la continuación del mismo programa desarrollado para 3ero y 4to año EGB, en el marco de dos proyectos de investigación consecutivos financiados por el gobierno de Chile (Fondecyt 1070851 y 1100391). El programa se sustenta sobre los postulados teóricos del aprendizaje socioemocional y considera la evidencia acumulada sobre la efectividad de intervenciones de este tipo a nivel nacional e internacional (DURLAK et al., 2011). Asimismo, el programa se sustenta sobre la evidencia recogida en diversas investigaciones cualitativas orientadas a conocer las expectativas y necesidades de los propios estudiantes y profesores 
en relación a este tema (BERGER et al., 2009, apud ALCALAY et al., 2012). De esta forma, el programa BASE se sustenta sobre dos aspectos conceptuales en relación al desarrollo infanto-juvenil: primero, adopta una perspectiva integral del desarrollo humano que considera como indisolubles lo cognitivo, afectivo, y ético. Segundo, pone especial énfasis en los vínculos interpersonales que se construyen en el contexto escolar, tanto profesor-alumno, entre alumnos, y de cada estudiante consigo mismo. Finalmente, también considera la importancia de la formación profesional docente que permita a los profesores atender a las necesidades socioemocionales de los estudiantes.

Las áreas del programa se orientan a favorecer en los estudiantes el conocimiento de sí mismo, la valoración de las competencias personales, la comprensión acerca del efecto de las emociones en los propios comportamientos y los de los otros y la autorregulación. También se considera como aspectos significativos dentro del programa la toma de perspectiva de los otros, las habilidades de comunicación, la relación con el mundo externo, la visión positiva del mundo, el afrontamiento pacífico de conflictos y las competencias prosociales. Se incluyó también en esta versión del programa una unidad sobre ética emocional, área que a la luz de la revisión bibliografía se encuentra poco trabajada pero es señalada por los autores como central (WEINER, 2006).

El programa fue diseñado para ser trabajado de manera integrada en el curriculum escolar de los cursos de 5to y 6to año EGB, idealmente con una periodicidad semanal (el programa contempla 12 unidades con dos actividades por unidad, para cada nivel). La modalidad del programa es la de un taller en el cual se pretende generar un espacio de conversación, reflexión personal y colectiva, y ejercitación de competencias socioemocionales en un espacio de contención afectiva. Para ello se diseñaron actividades que integran diferentes metodologías y modalidades de expresión (conversación, dramatizaciones, construcciones artísticas, integración de elementos extra-escolares) como también espacios de trabajo y reflexión individual, diádicas, y grupales, los cuales permiten distintos niveles de compromiso, intimidad y profundidad. Se propone que el programa sea implementado por profesores que tengan cercanía con el grupo curso (profesores jefe o tutores), de manera de generar un espacio de intimidad y seguridad afectiva. Para la adecuada implementación del programa se diseñó un libro para el profesor y/o monitor, que incluye una revisión teórica y conceptual, una descripción del programa, y todas las actividades tal como aparecen en el libro del estudiante con comentarios e indicaciones para aprovechar 
dichas actividades, posibles dificultades que podrían aparecer, y sugerencias de actividades complementarias. El programa también considera un libro del estudiante, el cual es de propiedad de cada uno y sirve como bitácora de su desarrollo a través de su participación en el programa.

\section{Metodología}

En el presente artículo se reporta el impacto de la implementación del programa BASE en distintos indicadores de bienestar socioemocional de estudiantes de 5to y 6to EGB. No obstante, es relevante señalar que el diseño y validación previos del programa siguieron los principios de la investigación-acción; en primer lugar se levantó información tanto de los programas existentes como de las características, percepciones, expectativas y necesidades de estudiantes y profesores sobre el aprendizaje y bienestar socioemocional. Luego se diseñaron las actividades por parte del equipo de investigación, y se contó también con la participación de un diseñador gráfico para la construcción de los manuales. El programa fue evaluado por jueces expertos en relación a sus contenidos y validez.

El impacto del programa fue evaluado con instrumentos cuantitativos, utilizando un diseño cuasi-experimental pre-post. Asimismo, se evaluó el proceso de implementación y la satisfacción del programa por parte de los profesores con metodologías cualitativas.

\section{Muestra}

El estudio se desarrolló en cinco instituciones educacionales de dependencia particular subvencionada de Santiago de Chile, e incluyó a todos los estudiantes de 5to y 6to año de EGB de dichas escuelas. La muestra total incluyó 848 participantes (46,8\% niños; $49,8 \%$ 5to año), distribuidos en 22 salas de clases. Tres escuelas fueron asignadas al grupo experimental donde se aplicó el programa BASE (16 salas de clases), mientras que las otras dos constituyeron el grupo control (6 salas). El diseño de la investigación fue de tipo cuasi-experimental con evaluación antes de iniciar la implementación y luego de ésta; la implementación del programa duró un período aproximado de siete meses. En ambas evaluaciones se solicitó a los participantes que reportaran sobre su bienestar socioemocional, su autoestima, su percepción sobre el clima escolar, y sobre las redes de pares al interior de sus cursos. Los profesores también reportaron sobre la autoestima de sus estudiantes. Se siguieron los procedimientos para resguardar a los participantes solicitando consentimiento parental informado y asentimiento informado de los 
estudiantes para participar, y se aseguró la confidencialidad y voluntariedad de la participación. Todos los procedimientos fueron aprobados por el comité de ética de la universidad patrocinante y la agencia que financió el proyecto. Se recolectaron datos completos para 694 estudiantes ( $82 \%$ de la muestra total).

\section{Instrumentos}

Bienestar socio-emocional. Se utilizó la escala de autoreporte de Bienestar SocioEmocional para evaluar esta dimensión. Esta escala fue desarrollada para la población chilena por Milicic, Arab, Alcalay, Berger \& Torretti (ver Arab, 2009) y constituye un instrumento de tamizaje para población escolar de entre 8 y 12 años. La escala incluye 52 itemes (algunos con puntuación reversa) con respuesta del tipo Likert de cuatro categorías ("me pasa todo el tiempo, me pasa usualmente, me pasa pocas veces, casi nunca me pasa). Presenta una alta consistencia interna $(\alpha=0,94)$. Algunos items son "me quedo solo en los recreos", "confío en que puedo alcanzar mis metas", "cuando tengo un problema encuentro maneras de resolverlo", "cuando estoy enojado sé como calmarme".

Autoestima. Esta variable fue medida a través de la Escala de Auto-Estima (TAE), desarrollada y validada en Chile por Marchant, Haeussler y Torretti (2002). Este instrumento establece una norma estadística respecto del nivel de autoestima y constituye un test de tamizaje para estudiantes de $3^{\circ}$ a $8^{\circ}$ año EGB que incluye una autoevaluación del propio estudiante, y una evaluación realizada por el profesor. La escala de autorreporte incluye 23 items de respuesta dicotómica (sí-no). La escala de reporte del profesor incluye 19 items con respuesta del tipo Likert de cuatro categorías.

Clima social escolar. Se utilizó la Escala de Clima Social, ECLIS (Arón et al., 2012). ECLIS incluye 82 itemes con respuestas del tipo Likert de cuatro alternativas (rango desde "siempre/todos" a "nunca/nadie". La escala incluye cuatro dimensiones: Los profesores (30 itemes, ejemplo: "son confiables", "se dan cuenta cuando uno tiene un problema"), los compañeros (15 itemes, ejemplo: "mis compañeros se ríen de mí", "saben como compartir"), el contexto escolar (10 itemes, ejemplo: "mi escuela es limpia y bien organizada", "tengo suficiente material de trabajo en mi sala"), y la satisfacción general con la escuela (27 itemes, ejemplo: "me siento orgulloso de mi escuela", "lo que me enseñan en clase me sirve"). La escala presenta para todas sus dimensiones alfas de Cronbach superiores a 0,72. 
Integración social. La integración social de los participantes fue evaluada a través de la metodología de Mapas Socio-Cognitivos (Social Cognitive Mapping, SCM), desarrollada por Cairns, Perrin y Cairns (1985). Los participantes reportaron sobre su grupo cercano de amigos y/o amigas dentro de su curso. Además, los participantes podían reportar otros grupos de compañeros que constituyan grupos en los cuales él o ella no participaran. La integración social es evaluada a través de las nominaciones recibidas como participante de un grupo por sobre el potencial máximo de nominaciones en cada curso.

\section{Resultados}

A nivel descriptivo, la Tabla 1 muestra las correlaciones entre las variables en estudio para el grupo experimental y para el grupo control. Tanto la integración social (para el grupo experimental) como la autoestima reportada por el profesor (para el grupo control) presentan asociaciones débiles e incluso no significativas con las otras variables; todas las otras variables del ámbito socioemocional correlacionan positivamente entre ellas para ambos grupos. Cabe destacar que se realizaron los mismos análisis de asociación por género, y solo se observó que la correlación entre autoestima percibida por el profesor y percepción de clima referida a los profesores y a los lugares fue significativa sólo para mujeres. No se observaron otras diferencias por género. Tampoco se observaron diferencias significativas por grado (5to y 6to) en las correlaciones presentadas.

Tabla 1 - Correlación entre las variables de estudio (evaluación pre) por grupo (experimental y control)

\begin{tabular}{|c|c|c|c|c|c|c|c|c|}
\hline & 1 & 2 & 3 & 4 & 5 & 6 & 7 & 8 \\
\hline 1. ASE & - & $.34^{\star \star}$ & $.30 * \star$ & $.50^{\star \star}$ & $.57^{\star \star}$ & $.39^{\star *}$ & $.52^{\star \star}$ & $.35^{\star *}$ \\
\hline 2. TAE - Alumno & $.55^{\star *}$ & - & $.34^{* *}$ & $.36^{* \star}$ & $.52^{\star *}$ & $.26^{* *}$ & $.30^{\star \star}$ & $.31^{* *}$ \\
\hline 3. TAE - Profesor & $.29^{\star *}$ & $.21^{* *}$ & - & .05 & $.16^{\star}$ & -.06 & .05 & $.22^{* *}$ \\
\hline $\begin{array}{l}\text { 4. ECLIS profe- } \\
\text { sores }\end{array}$ & $.38^{\star \star}$ & $.25^{\star *}$ & $.13^{* *}$ & - & $.45^{\star \star}$ & $.62^{\star \star}$ & $.58^{\star \star}$ & $.16^{*}$ \\
\hline 5. ECLIS pares & $.62^{* *}$ & $.51^{\star *}$ & $.16^{\star \star}$ & $.36^{* \star}$ & - & $.39^{\star *}$ & $.44^{\star *}$ & $.24^{* *}$ \\
\hline
\end{tabular}




\begin{tabular}{|c|c|c|c|c|c|c|c|c|}
\hline 6. ECLIS contexto & $.46^{\star *}$ & $.30^{* *}$ & $.19^{* *}$ & $.49^{\star *}$ & $.37^{\star \star}$ & - & $.59^{\star *}$ & .04 \\
\hline 7. ECLIS escuela & $.50 * \star$ & $.23^{* *}$ & $.10^{*}$ & $.53^{\star \star}$ & $.35^{\star *}$ & $.53^{\star \star}$ & - & .09 \\
\hline $\begin{array}{l}\text { 8. Integración } \\
\text { social }\end{array}$ & $.25^{\star *}$ & $.27^{\star *}$ & $.29 * *$ & -.03 & $.28^{* *}$ & .04 & .01 & - \\
\hline
\end{tabular}

Nota: ${ }^{* *} p<.01 ;{ }^{*} p<.05$. Correlaciones para el grupo experimental bajo la diagonal, para el grupo control sobre la diagonal. ASE = Bienestar socioemocional; TAE = Test de Autoestima; ECLIS = Escala de Clima Escolar

Se realizó una comparación en la evaluación entre el grupo experimental y control en todas las variables de interés. Los participantes del grupo experimental presentaron una mejor percepción del clima escolar en todas sus dimensiones ( $t \mathrm{~s}=3,03,3,81,3,38$ y $6,01, p \mathrm{~s}<0,01)$, y por el contrario fueron percibidos por sus profesores como teniendo autoestima más baja $(t=3,64, p<0,01)$. Mismas comparaciones por género mostraron que las mujeres presentaron una percepción del clima escolar referida a los contextos más positiva que los hombres $(\mathrm{t}$ $\left.=2,47^{*}\right)$, mientras que los hombres presentaron mayores indicadores de integración social $\left(\mathrm{t}=2,33^{*}\right)$. En la segunda medición, además de estas diferencias se observó una mayor autoestima reportada por las estudiantes mujeres $(\mathrm{t}=3,31 * *)$.

La Tabla 2 presenta los promedios en las evaluaciones pre y post de los grupos experimental y control en todos los indicadores, señalando además diferencias significativas entre ambas mediciones (pruebas t para muestras relacionadas), y como efecto de la interacción de ambos factores (Anova para medidas repetidas, tiempo como factor intrasujeto, condición experimental o control como factor intersujeto). Como puede observarse, los estudiantes que participaron del programa aumentaron su autoestima (tanto percibida por ellos mismos como por sus profesores) $(t \mathrm{~s}=60,41 \mathrm{y}$ $15,53, p \mathrm{~s}<0,01)$, mejoraron su percepción del clima escolar en relación a sus profesores $(t=3,07, p<0,01)$, sus pares $(t=4,34, p<0,01), \mathrm{y}$ mejoraron su integración social $(t=4,84, p<0,01)$. En comparación, el grupo control sólo mejoró su auto percepción de autoestima $(t=23,72, p<$ $0,01)$, pero disminuyó en su percepción de clima social escolar en distintas dimensiones y su integración social $(t \mathrm{~s}=5,23,3,93$ y 4,70 respectivamente, $p$ s $<0,01)$. En otras palabras, participar del programa BASE tuvo por una 
parte un impacto positivo en distintas variables, pero además actuó como factor protector frente al descenso en distintos indicadores observados en el grupo control (que puede deberse a los periodos del año en que se realiza la medición) y no observados en los participantes del programa.

Tabla 2 - Promedios y estadísticos de los grupos experimental y control en evaluaciones pre y post

\begin{tabular}{|c|c|c|c|c|c|c|c|}
\hline & $\begin{array}{l}\text { Experimental } \\
\quad(n=610)\end{array}$ & & $\begin{array}{l}\text { Control } \\
(n=238)\end{array}$ & Interacción & & & \\
\hline & pre & Post & $\mathrm{t}$ & pre & post & $\mathrm{T}$ & $\mathrm{F}$ \\
\hline ASE & 3,27 & 3,28 & $<1$ & 3,22 & 3,18 & $-1,45$ & $4,76^{*}$ \\
\hline TAE-Alumno & 19,01 & 32,42 & $60.41^{\star *}$ & 18,48 & 30,33 & $23,72^{* *}$ & $12,29 * *$ \\
\hline TAE-Profesor & 56,16 & 62,48 & $15.53^{\star \star}$ & 59,20 & 58,78 & $<1$ & $50,63^{* *}$ \\
\hline $\begin{array}{l}\text { ECLIS } \\
\text { profesores }\end{array}$ & 3,03 & 2,98 & $3,07^{* *}$ & 2,93 & 2,90 & $-1,38$ & $<1$ \\
\hline ECLIS pares & 3,03 & 3,11 & $4,34 * *$ & 2,85 & 2,85 & $<1$ & $6,86^{\star *}$ \\
\hline $\begin{array}{l}\text { ECLIS } \\
\text { contexto }\end{array}$ & 3,07 & 2,96 & $-4,76^{\star \star}$ & 2,91 & 2,71 & $-5,23^{\star \star}$ & $9,02^{* *}$ \\
\hline $\begin{array}{l}\text { ECLIS } \\
\text { colegio }\end{array}$ & 3,22 & 3,29 & $<1$ & 3,02 & 2,89 & $-3,93^{* *}$ & $<1$ \\
\hline $\begin{array}{l}\text { Integración } \\
\text { social }\end{array}$ & 14,40 & 15,91 & $4,84^{* *}$ & 14,40 & 12,34 & $-4,70^{\star *}$ & $44,58^{\star *}$ \\
\hline
\end{tabular}

Nota: ${ }^{*} p<0,05 ;{ }^{* *} p<0,01$. ASE $=$ Bienestar socioemocional; TAE = Test de Autoestima; ECLIS $=$ Escala de Clima Escolar

Se evaluó también el efecto de la interacción entre ambos factores (grupo experimental o control, y evaluación pre y post), observando un claro efecto sobre el bienestar socioemocional $(\mathrm{F}=4,76, \mathrm{p}<0,05)$, ambas mediciones de la autoestima ( Fs $=12,29$ y 50,63, ps $<0,01)$, dimensiones del clima escolar $(\mathrm{Fs}=6,86$ y 9,02, $\mathrm{ps}<0,01)$ e integración social $(\mathrm{F}=44,58, \mathrm{p}$ $<0,01)$. A manera de ejemplo las figuras 1 y 2 muestran gráficamente el impacto de la participación del programa en la autoestima percibida por el profesor y en la integración social, respectivamente. 




Figura 1 - Efecto sobre la Autoestima percibida por el profesor (TAEP) de la interacción entre participar o no del programa y el tiempo entre la evaluación pre y post.

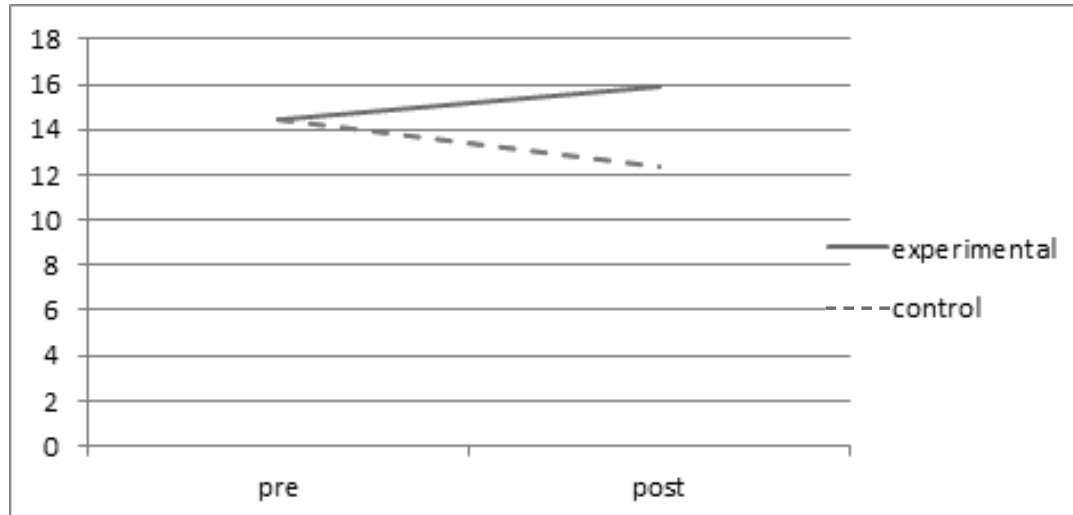

Figura 2 - Efecto sobre la Integración social de la interacción entre participar o no del programa y el tiempo entre la evaluación pre y post

Por otra parte, los mismos análisis se llevaron a cabo por separado para estudiantes de quinto y sexto año de enseñanza general básica. Si bien en ambos grupos se observó la misma tendencia, los niveles de significancia fueron distintos debido a los grados de libertad y poder de las pruebas estadísticas. Para 5to se observaron efectos de la interacción tiempo x condición para el bienestar socioemocional, la 
autoestima reportada por el profesor, la percepción del clima escolar referido a pares y lugares, y la integración social, mientras que para 6to se observó también un efecto en la autoestima reportada por los propios estudiantes, mientras que no se observó el efecto sobre el bienestar socioemocional.

El género fue también considerado un factor importante a evaluar. Cabe mencionar que antes de iniciar el programa y al agregar el total de la muestra $(n=848)$, se observó que las mujeres presentaron una percepción del clima escolar referida a los lugares más positiva que los hombres $(\mathrm{t}=2,47, \mathrm{p}<0,05)$, mientras que los hombres presentaron mayores indicadores de integración social $(\mathrm{t}=2,33, \mathrm{p}<$ $0,05)$. En la segunda medición, además de estas diferencias se observó una mayor autoestima reportada por las estudiantes mujeres $(\mathrm{t}=3,31, \mathrm{p}<0,01)$. Respecto de los efectos del programa, las pruebas de interacción (Anova de medidas repetidas) por género mostraron que los efectos sobre el bienestar socioemocional, la autoestima reportada por las mujeres y la percepción del clima escolar referido a los lugares fueron significativos para las mujeres pero no para los hombres.

\section{Discusión}

Los modelos educativos y políticas públicas en distintos países, si bien en los últimos años han sido explícitos en declarar la importancia de la dimensión socioemocional en la educación, han continuado enfatizando los logros académicos e indicadores relacionados a ellos como única fuente de validación de la calidad. Sin embargo, diversos autores y estudios recientes muestran un nexo indisoluble entre ambas dimensiones (BERGER et al., 2011; DURLAK et al., 2011; HAWKINS et al., 2004), nexo que también se refleja en los resultados presentados en este estudio. Potenciar el bienestar y aprendizaje socioemocional de los estudiantes redunda también en mayores logros académicos, a través de la generación de ambientes nutritivos, el desarrollo de competencias necesarias para aprovechar la experiencia académica, el desarrollo de una relación positiva entre estudiantes y docentes y el fortalecimiento de la autoestima.

La perspectiva anterior se ha visto fuertemente impulsada por la generación de nichos académicos en distintos países (por ejemplo, Collaborative for Academic, Social, and Emotional Learning - CASEL — en Estados Unidos, y Grup de Recerca en Orientació Psicopedagògica-GROP—en España), los cuales han fomentado el desarrollo y evaluación sistemática de programas de intervención 
tendientes a fortalecer el desarrollo socioemocional. Esto ha permitido también conocer aquellos factores que se asocian al mayor éxito de dichos programas, entre ellos, la articulación de los programas a la manera de un currículo, la participación de la comunidad educativa, la integración de aspectos teóricos y prácticos, y la claridad y explicitación en sus objetivos (DURLAK et al., 2011).

Es precisamente a la luz de esta evidencia que se presenta la experiencia del programa BASE, el cual se sustenta sobre estos pilares y considera la necesidad de un proceso formativo docente respecto de su propio aprendizaje socioemocional. Los positivos resultados alcanzados por este programa permiten suponer que su fundamentación conceptual, diseño e implementación debieran ser considerados como factores relevantes en toda intervención educativa en esta línea. Sin embargo, la necesidad de considerar las posibilidades reales que brinda el sistema educativo y las demandas consideradas críticas por el mismo hace evidente la tensión existente entre dicho contexto y la generación e implementación de programas de este tipo. BASE fue implementado como un programa autosustentado, que incluyó una jornada de capacitación inicial y un monitoreo a distancia, pero no un proceso de monitoreo o capacitación constante. El reporte de los profesores participantes da cuenta de que en el programa ellos se hicieron parte de las comunidades curso junto a sus estudiantes. Esto constituye un elemento esencial, en la medida que el programa no es percibido como una amenaza a la labor docente, sino por el contrario, permite favorecer los vínculos al interior de la sala de clases, lo cual es esencial para la generación de ambientes de aprendizaje para el desarrollo y éxito de los estudiantes (JENNINGS; GREENBERG, 2009).

Coherente con la noción de apego escolar desarrollada en el marco teórico de este estudio, estos vínculos son centrales pues los profesores en esta etapa se constituyen como figuras de apego secundario, y de manera general toda la experiencia escolar se constituye como un espacio de seguridad, aceptación y contención. En este sentido, el fortalecimiento de vínculos sanos y nutritivos debiera ser un objetivo prioritario en cualquier iniciativa educacional (EXTREMERA; FERNÁNDEZ-BERROCAL, 2003; BERGIN; BERGIN, 2009), lo que permitiría a los establecimientos educacionales contar con un marco para prevenir problemas, pero aún más importante para promover el bienestar de sus estudiantes a través de la valoración de la diversidad, de las diferencias, y el énfasis en el conocimiento y respeto por el otro. 
Considerando lo anterior, y a la luz de la evidencia del impacto del programa BASE en el bienestar de los estudiantes que viene a confirmar los consistentes datos reportados en la literatura internacional, se hace imprescindible que las políticas públicas en educación comiencen a intencionar, de manera clara y explícita, una perspectiva integral del desarrollo que considere la dimensión socioemocional de los estudiantes, pero que también enfatice una perspectiva colectiva de lo socioafectivo cuidando tanto el bienestar de los profesores como el fortalecimiento de los contextos sociales nutritivos. Esto permitiría construir una sociedad justa, inclusiva, tolerante y respetuosa, y responsable por el bienestar de todos sus miembros.

\section{Referencias}

ABBOTT, R. D. et al. Changing teaching practices to promote achievement and bonding to school. American Journal of Orthopsychiatry, [S. 1.], n. 68, p. 542-552, 1998.

ALCALAY, L. et al. Aprendizaje socioemocional y apego escolar: favoreciendo la educación en diversidad. In: . Diversidad y educación: miradas desde la psicología educacional. Santiago: Ediciones Universidad Católica, 2012. p. 5-68.

ÁlVAREZ, M. (Coord.). Diseño y evaluación de programas de educación emocional. Barcelona: Ciss-Praxis, 2001.

ARAB, M. P. Diseño y construcción de una escala de autoreporte de bienestar socioemocional para niños de 8-12 años de EGB. 2009. $152 \mathrm{f}$. Tese (Doutorado)-Psicología Educacional, Pontificia Universidad Católica de Chile, Santiago, 2009.

ARÓN, A. M.; MILICIC, N. Clima social escolar y desarrollo personal: un programa de mejoramiento. Santiago: Editorial Andrés Bello, 1999.

ARÓN, A.; MILICIC, N.; ARMIJO, I. Clima social escolar: una escala de evaluación: escala de clima social escolar, Universitas Psychologica, [S.1.], v. 11, n. 3, p. 803-813, 2012. 
ASHDOWN, D.; BERNARD, M. Can explicit instruction in social and emotional learning skills benfit the social-emotional development, well-being, and academic achievement of young children?. Early Childhood Education Journal, [S.1.], n. 39, p. 397-405, 2012.

BERGER, C. et al. Socioemotional wellbeing and academic achievement: evidence from a multilevel approach. Psicologia: reflexao e critica, Porto Alegre, v. 24, p. 344-351, 2011.

BERGER, C. et al. Socio-emocional en contextos escolares: La percepción de estudiantes chilenos. Estudios sobre Educación, [S.1.], n. 17, p. 21-43, 2009.

BERGIN, C.; BERGIN, D. Attachment in the classroom. Educational Psychology Review, [S.1.], n. 21, p. 141-170, 2009.

BIRCH, S. H.; LADD, G. W. Children's interpersonal behaviors and the teacher child relationship. Developmental Psychology, [S.1.], n. 34, p. 934 946, 1998.

BISQUERRA, R.; PÉREZ, N. Las competencias emocionales. Educación $X X 1$, [S.1.], n. 10, p. 61-82, 2007.

CAIRNS, R., PERRIN, J.; CAIRNS, B. Social structure and social cognition on early adolescence: affiliative patterns. Journal of Early Adolescence, [S.1.], n. 5, p. 339-355, 1985.

CHAUX, E.; LLERAS, J.; VELASQUEZ, A. Competencias ciudadanas: de los estándares al aula. Bogotá: Ediciones Uniandes, 2004.

CHEN, X. et al. Effects of the peer group on the development of social functioning and academic achievement: a longitudinal study in Chinese children. Child development, v. 79, n. 2, p. 235-51, 2008.

DURLAK, J. A.; WEISSBERG, R. P. The impact of after-school programs that seek to promote personal and social skills. Casel: University of Illinois at Chicago, 2007. Diponível em: <http://www.casel.org/downloads/ASP-Full. pdf $>$. Aceso em: 21 ago. 2013. 
DURLAK, J. A., WEISSBERG, R. P.; PACHAN, M. A meta-analysis of after-school programs that seek to promote personal and social skills in children and adolescents. American Journal of Community Psychology, [S.1.], n. 45, p. 294-309, 2010.

DURLAK, J. A. et al. The impact of enhancing students' social and emotional learning: A meta-analysis of school-based universal interventions. Child Development, [S.1.], n. 82, p. 405-432, 2011.

EISENBERG, N. Introduction. In: DAMON, W.; R. LERNER, R.; EISENBERG, N. (Ed.). Handbook of child psychology: vol. 3: social, emotional and personality development. 6. ed. New York: Wiley, 2006. p. 1-23.

EISENBERG, A.; GARVEY, C. Children use of verbal strategies in resolving conflicts. Discourse Processes, [S.1.], n. 4, p. 149-170, 1981.

EXTREMERA, N.; FERNÁNDEZ-BERROCAL, P. La inteligencia emocional: métodos de evaluación en el aula. Revista Iberoamericana de Educación, [S.1.], n. 30, p. 1-12, 2003.

FLETCHER, A.; HUNTER, A.; EANES, A. Links between social network closure and child well-being: the organizing role of friendship context. Developmental Psychology, [S.1.], n. 42, p. 1057-1068, 2006.

FRENZEL, A. C. et al. Emotional transmission in the classroom: Exploring the relationship between teacher and student enjoyment. Journal of Educational Psychology, [S.1.], v. 3, n. 101, p. 705-716, 2009.

GOLDENBERG, I.; MATHESON, K.; MANTLER, J. The assessment of emotional intelligence: a comparison of performance-based and self-report methodologies. Journal of Personality Assessment, [S.1.], v. 86, p. 33-45, 2006.

GOLEMAN, D. La inteligencia emocional. Buenos Aires: Javier Vergara Editor S.A., 1996.

GRESHAM, F.; ELLIOTT, S. Assessment and classification of children's social skills: a review of methods and issues. School Psychology Review, [S.1.], n. 13, p. 292-301, 1984. 
GUERRA, N.; BRADSHAW, C. Linking the prevention of problem behaviors and positive youth development: core competencies for positive youth development and risk prevention. New Directions for Child and Adolescent Development, [S.1.], n. 122, p. 1-17, 2008.

HAEUSSLER, I. M.; MILICIC, N. Confiar en uno mismo. Santiago: Ed. Dolmen, 1995.

HAMM, J. V. et al. A. Teachers' attunement to students' peer group affiliations as a source of improved student experiences of the school socialaffective context following the middle school transition. Journal of Applied Developmental Psychology, [S.1.], n. 32, p. 276-286, 2011.

HAMRE, B.; PIANTA, R. C. Early teacher-child relationships and trajectory of school outcomes through eighth grade. Child Development, [S.1.], n. 72, p. 625-638, 2001.

HAMRE, B.; PIANTA, R. C. Student-teacher relationships. In: BEAR, G.; MINKE, K. M. (Ed.), Children's needs III: development, prevention, and intervention. Bethesda, MD: NASP, 2006. p. 59-72.

HAWKINS, J . D et al. Preventing adolescent health-risk behaviors by strengthening protection during childhood. Archives of Pediatrics and Adolescent Medicine, [S.1.], n. 153, p. 226-234, 1999.

HAWKINS, J. D. et al. Social development and social and emotional learning. In: ZINS, J. E.; WEISSBERG, R. P.; WANG, M. C.; WALBERG, H. J. (Ed.). Building academic success on social and emotional learning. NNew York: Teacher's College Press, 2004. p. 135-151.

HOFFMAN, D. M. Reflecting on Social Emotional Learning: a critical perspective on trends in the united states. Review of Educational Research, [S. 1.], v. 79, n. 2, p. 533-556, 2009.

HUBBARD, J.; COIE, J. Emotional correlates of social competence in children's peer relationships. Merrill-Palmer Quarterly, [S. 1.], n. 40, p. 1-20, 1994. 
JENNINGS, P.; GREENBERG, M. The prosocial classroom: teacher social and emotional competence in relation to student and classroom outcomes. Review of Educational Research, [S.1.], n. 79, p. 491-525, 2009.

KORNBLIT, A.; ADASZKO, D; DILEO, P. Clima social escolar y violencia: un vínculo explicativo posible. Un estudio en escuelas medias argentinas. In: BERGE, C.; LISBOA, C. (Ed.). Violencia escolar: estudios y posibilidades de intervención en Latinoamérica. Santiago: Universitaria, 2008. p. 109-138.

KUPERMINC, G.; LEADBEATER, B; BLATT, S. School social climate and individual differences in vulnerability to psychopathology among middle school students. Journal of School Psychology, [S.1], n. 39, p. 141-159, 2001.

LAURSEN, B.; FINKELSTEIN, B; TOWNSENDS, N. A developmental meta-analysis of peer conflict resolution. Developmental Review, [S.1], v. 21, n. 4, p. 423-449, 2001.

LOPES, P. et al. Emotion regulation abilities and the quality of social interaction. Emotion, [S.1.], n. 5, p. 113-118, 2005.

LOUKAS, A.; ROBINSON, S. Examining the moderating role of perceived school climate in early adolescent adjustment. Journal of Research on Adolescence, [S.1.], v. 14, n. 2, p. 209-233, 2004.

LUCKNER, A.; PIANTA, R. Teacher-student interactions in fifth grade classrooms: relations with children's peer behavior. Journal of Applied Developmental Psychology, [S.1.], n. 32, p. 257-266, 2011.

MALECKI, C. K.; ELLIOT, S. N. Children's social behaviors as predictors of academic achievement: A longitudinal analysis. School Psychology Quarterly, [S.1.], n. 17, p. 1-23, 2002.

MARCHANT, T.; HAEUSSLER, I. M.; TORRETTI, A. TAE: Batería de tests de autoestima escolar. Santiago: Ediciones Universidad Católica de Chile, 2002. 
MURRAY, C.; GREENBERG, M. T. Children's relationship with teachers and bonds with school: An investigation of patterns and correlates in middle childhood. Journal of School Psychology, [S.1.], n. 38, p. 423-445, 2000.

OJANEN, T., GRÖNROOS, M.; SALMIVALLI, C. An interpersonal circumplex model of children's social goals: links with peer-reported behavior and sociometric status. Developmental psychology, [S.1.], v. 41, n. 5, p. 699$710,2005$.

OSHER, D. et al. A comprehensive approach to promoting social, emotional, and academic growth in contemporary schools. In: THOMAS, A.; J. GRIMES, J. (Ed.). Best practices in school psychology. 5 ed. Bethesda, MD: National Association of School Psychologists, 2007. v. 5, p. 1263-1278.

PAYTON J. M. et. al. Social and emotional learning: a framework for promoting mental health and reducing risk behaviors in children and youth. Journal of School Health. [S.1.], v. 70, n. 5, p. 179-185, 2000.

PIANTA, R. C.; HAMRE, B.; STUHLMAN, M. Relationships between teachers and children. In: REYNOLDS, W. M.; MILLER, G. E. (Ed.). Comprehensive handbook of psychology. New York: Wiley, 2003. v. 7, p. 199-234.

POTOCNJAK, M.; BERGER, C.; TOMICIC, T. Una aproximación relacional a la violencia escolar entre pares en adolescentes chilenos: perspectiva adolescente de los factores intervinientes. Psykhe, [S.1.], n. 20, p. 39-52, 2011.

RIVERA, M.; MILICIC, N. Alianza Familia-Escuela: percepciones, creencias, expectativas y aspiraciones de padres y profesores de enseñanza general básica. Psykhe, [S.1.], n. 15, p. 119-135, 2005.

ROTH, G. et al. Autonomous motivation for teaching: how self-determined teaching may lead to self-determined learning. Journal of Educational Psychology, v. 99, n. 4, p. 761-774, 2007.

SALOVEY, P.; MAYER, J. Emotional Intelligence: imagination, cognition and personality, [S.1.], v. 9, n. 3, p. 185-211, 1990. 
SMITH, P.; ANANIADOU, K.; COWIE, H. Interventions to reduce school bullying. Canadian Journal of Psychiatry, [S.1.], n. 48, p. 591-599, 2003.

STIPEK, D.; MILES, S. Effects of aggression on achievement: does conflict with the teacher make it worse?. Child Development, [S.1.], n. 79, p. 1721$1735,2008$.

WEINER, B. Social motivation: justice and the moral emotions. Mahwah: Lawrence Erlbaum, 2006.

ZINS, J. et al. (Ed.). Building academic success on social and emotional learning. New York: Teacher's College Press, 2004.

Recebido em: 26/03/2013

Aceito para publicação em: 27/08/2013

\section{Aprendizagem socioemocional em estudantes de quinta e sexta série: apresentação e avaliação de impacto do programa BASE}

\section{Resumo}

O trabalho apresenta o desenvolvimento e a avaliação de impacto de um programa para favorecer o bem-estar e a aprendizagem socioemocional em estudantes de quinta e sexta série de Ensino Fundamental: o programa BASE. Por meio de uma revisão teórica da aprendizagem socioemocional se fundamenta a ênfase do programa no desenvolvimento de vínculos interpessoais de qualidade e o desenvolvimento das competências necessárias, integrando fatores de desenvolvimento individual e do contexto que favorece o mencionado desenvolvimento, assim como a geração de comunidades sadias e nutritivas. Para avaliar o impacto do programa foi utilizado um desenho quase-experimental do tipo pre-post com grupo controle. Os resultados mostraram um impacto significativo positivo do programa na autoestima, integração social, e na percepção do clima escolar dos estudantes que participaram, quando comparados com o grupo controle. Foram discutidos os resultados do programa e suas possíveis implicações para o desenvolvimento de intervenções nesse campo.

Palavras-chave: Aprendizagem socioemocional. Bem-estar. Ensino Fundamental. 


\section{Socioemotional learning in students of fifith and sixth grades: presentation and impact evaluation of BASE program}

\section{Abstract}

This article presents the development and impact evaluation of a program to enhance the wellbeing and socioemotional learning of 5th and 6th graders: The BASE program. Based on the theoretical framework of socioemotional learning, the emphasis of the program is oriented towards fostering quality interpersonal bounds and the development of competencies that are necessary for this, integrating both individual and contextual factors and the generation of healthy and nurturing communities. The study features a quasi-experimental pre-post design with a control group in order to assess the impact of the program. Results show a significant positive effect on self-esteem, social integration, and the perception of the school social climate of students who were part of the program, compared to their counterparts in the control group. These results are discussed in light of their potential implications for future educational interventions.

Keywords: Socioemotional learning. Wellbeing. Primary school.

Este trabajo ha sido financiado en parte por el Fondo Nacional de Desarrollo Científico y Tecnológico de Chile, FONDECYT, proyecto 1100391, y por el Centro Interdisciplinario de Estudios Interculturales e Indígenas-ICIIS, Código de Proyecto: CONICYT/FONDAP/15110006 